\title{
CMOS Duobinary Transceiver for Multigigabit Communications
}

\author{
J. Aguirre ${ }^{1}$, C. Sánchez-Azqueta ${ }^{1}$, E. Guerrero ${ }^{1}$, C. Gimeno ${ }^{2}$, S. Celma ${ }^{1}$ \\ ${ }^{1}$ Grupo de Diseño Electrónico \\ Instituto de Investigación en Ingeniería de Aragón (I3A) \\ Universidad de Zaragoza, Mariano Esquillor s/n, 50018, Zaragoza, Spain. \\ Tel. +34-976762707, e-mail: jag@unizar.es \\ ${ }^{2}$ ICTEAM Institute, Université Catholique de Louvain, Louvain-la-Neuve, Belgium
}

\section{Abstract}

This work presents a CMOS transceiver for amplitude duobinary modulation over an equalized 50-m step-index plastic optical fiber (SI-POF). Both duobinary precoder and decoder have been fabricated in $0.18-\mu \mathrm{m}$ CMOS technology, enabling a bandlimited system of $700 \mathrm{MHz}$ to operate at $3.125 \mathrm{Gbps}$ with a consumption of $28.4 \mathrm{~mW}$.

\section{Introduction}

Optical fibers have emerged as an interesting option instead of copper as transmission channel in shortrange communications due to their excellent mechanical features. SI-POF is lightweight, compact, robust when bent and its large core makes it easy to handle and install. However, some kind of signal correction is necessary to compensate its narrow bandwidth-length product of $45 \mathrm{MHz} \times 100 \mathrm{~m}$, in order to enable SI-POF for multigigabit applications.

An interesting possibility in this direction is the use of data formats different from traditional non-return-to-zero (NRZ) modulation. Multilevel modulation allows an increase of the data rate at the expense of design complexity of both emitter and receiver, as well as signal-to-noise ratio (SNR) degradation. In this way, the choice of duobinary modulation is a very good option. It is a modulation format that doubles the data rate with respect to NRZ by simple and low-power circuitry, without suffering as much SNR penalty as other amplitude modulation formats, like 4-PAM [1].

\section{Duobinary Technique}

Inter-symbol interference (ISI) is an overlapping in time between consecutive transmitted bits that happens when the spectral components of the transmitted signal are above the cut-off frequency of the channel. Duobinary modulation takes advantage of ISI, assuming that all the received symbols suffer from it.
Fig. 1 shows the block diagram of the amplitude duobinary transmission system. A precoder processes a bit sequence and the clock signal according to $\operatorname{PRE}(\mathrm{t})=\operatorname{PRE}\left(\mathrm{t}-\mathrm{T}_{\mathrm{b}}\right) \oplus \mathrm{IN}(\mathrm{t})$, where $\mathrm{T}_{\mathrm{b}}$ is the bit period and $\oplus$ denotes the logic XOR operation. The output of the precoder, PRE, is also NRZ modulated and it is sent through a bandlimited channel and a front-end. Due to ISI, the front-end output will be a distorted signal DUO, duobinary modulated, $\operatorname{DUO}(\mathrm{t})=1 / 2\left[\operatorname{PRE}(\mathrm{t})+\operatorname{PRE}\left(\mathrm{t}-\mathrm{T}_{\mathrm{b}}\right)\right]$. Finally, signal DUO, which is a 3-level signal, must be decoded in the receiver to recover the original bit sequence. Table I contains the truth table of this decoding process $\Psi$ : DEC $(\mathrm{t})=\Psi[\mathrm{DUO}(\mathrm{t})]=\mathrm{IN}(\mathrm{t})$.

An important feature of the proposed duobinary sequence is that it is correlated and hence not all transitions can occur. The result is that a duobinary signal has a power spectral density (PSD) equal than that of an NRZ signal with half the data rate [2].

\section{Circuit description}

The precoder box in Fig. 1 shows the block diagram of the implemented precoder. The data signal and the clock signal enter a two-input AND logic gate, whose output goes to a Flip Flop (FF) working as a frequency divider. The FF is formed by two D-latches in master-slave configuration with negative feedback. All the cells in this work have been implemented using SCL cells, achieving 30\% higher data rate in comparison to CMOS static logic.

Table 1. Duobinary Truth Table.

\begin{tabular}{|c|c|c|c|c|}
\hline IN(t) & PRE(t-T & PRE(t) & DUO(t) & DEC(t) \\
\hline \multirow{2}{*}{0} & 0 & 0 & 0 & \multirow{2}{*}{0} \\
\cline { 2 - 4 } & 1 & 1 & 1 & \\
\hline \multirow{2}{*}{1} & 0 & 1 & $1 / 2$ & \multirow{2}{*}{1} \\
\cline { 2 - 4 } & 1 & 0 & & \\
\hline
\end{tabular}


The channel is a $50-\mathrm{m}$ Mitsubishi GH SI-POF, whereas the front-end is the combination of a large area Si PIN photodetector, a transimpedance amplifier and a continuous-time equalizer [3]. It all results in a system with $700 \mathrm{MHz}$ bandwidth and $100 \mathrm{~dB} /$ decade roll-off. The effect of the SI-POF channel properly equalized has been simulated by means of a $5^{\text {th }}$ order low-pass Bessel filter with $700 \mathrm{MHz}$ bandwidth.

The duobinary decoder has been implemented with two voltage comparators and an XOR gate. The block diagram is shown in the decoder box of Fig. 1. The two voltage comparators have the same structure and they have been implemented with three differential amplifiers connected in cascade. The voltage $r e f_{\mathrm{L}}$ in the first comparator must be adjusted to be between the logic levels 0 and $1 / 2$ of the DUO signal, whereas $\operatorname{ref}_{\mathrm{H}}$ in the second one must be adjusted between the corresponding $1 / 2$ and 1 levels.

\section{Results}

The proposed duobinary precoder and decoder have been designed in a standard $0.18-\mu \mathrm{m}$ CMOS technology fed with $1.8 \mathrm{~V}$ single supply. The precoder consumes $10.3 \mathrm{~mW}$ and the decoder $18.1 \mathrm{~mW}$. The designs are tested for $3.125 \mathrm{Gbps}$ with a pseudo-random bit sequence (PRBS) of $2^{31}-1$ bits. Fig. 2 shows the active zone of the dies. Fig. 3 shows the eye diagrams of the main parts of the transceiver: The precoder output, signal PRE; the TIA output (before equalization); the equalizer output, signal DUO; and finally, the decoder output, signal DEC. This output eye diagram presents an rms jitter of $3.6 \mathrm{ps}$ and an aperture of $185 \mathrm{mV}$.

As it can be seen, a data rate of $3.125 \mathrm{Gbps}$ can be achieved with a bandwidth of only $700 \mathrm{MHz}$. So, the equalization requirements of duobinary are relaxed in comparison with NRZ, which also alleviates power consumption of the equalizer and reduces the pernicious high-frequency noise.

\section{Conclusion}

This paper presents a novel CMOS transceiver that enables an equalized 50-m SI-POF channel to target $3.125 \mathrm{Gbps}$ in a system limited in bandwidth at $700 \mathrm{MHz}$ by means of amplitude duobinary modulation. It is designed with high-frequency cells implemented in $0.18-\mu \mathrm{m}$ CMOS technology fed with $1.8 \mathrm{~V}$, presenting a total consumption of only $28.4 \mathrm{~mW}$.

\section{REFERENCES}

[1]. ZEOLLA, D., NESPOLA, A. and GAUDINO, R Comparison of different modulation formats for 1$\mathrm{Gb} / \mathrm{s}$ SI-POF transmission systems. IEEE Photonic Technology Letters. 2011, 23(14), 950-952.

[2]. AgUiRRE, J. et al. Precoder and decoder for duobinary modulation over equalized 50-m SI-POF. Proceedings of the Conference on PhD Research in Microelectronics and Electronics (PRIME), Lisbon: IEEE 2016. Accepted.

[3]. GIMENO, C. et al. A 1-V 1.25-Gbps CMOS analog front-end for short reach optical links. Proceedings of the European Solid-State Circuits Conference (ESSCIRC), Bucharest: IEEE 2013, pp. 339-342.

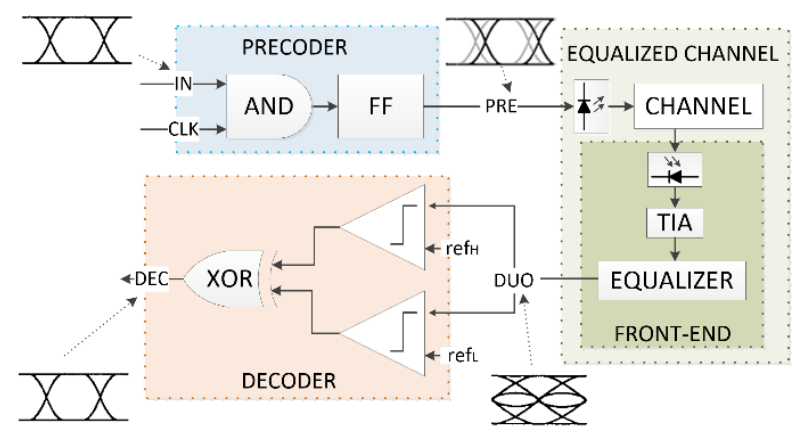

Fig. 1. Block diagram of the proposed duobinary system.

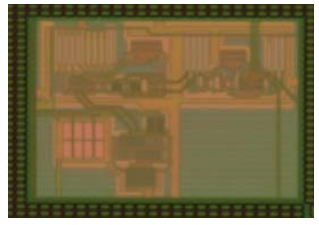

(a)

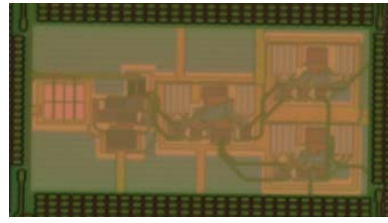

(b)
Fig. 2. Active zone of the fabricated ICs. (a) Duobinary precoder $(140 \mu \mathrm{m} \times 95 \mu \mathrm{m})$ and (b) duobinary decoder (187 $\mu \mathrm{m} \times 89 \mu \mathrm{m})$.

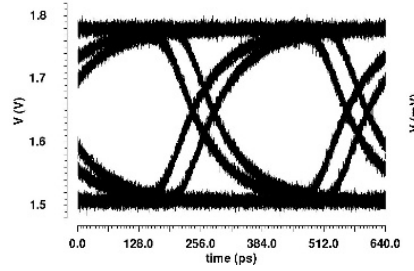

(a)

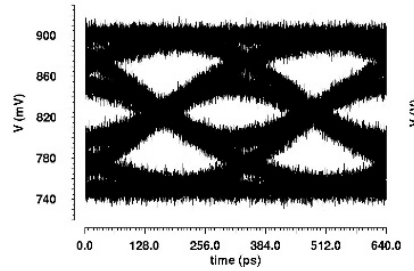

(c)

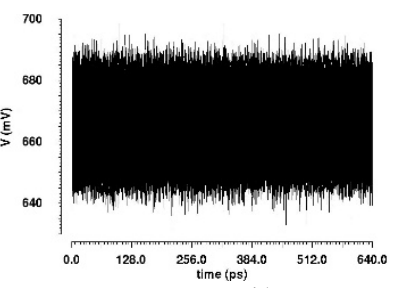

(b)

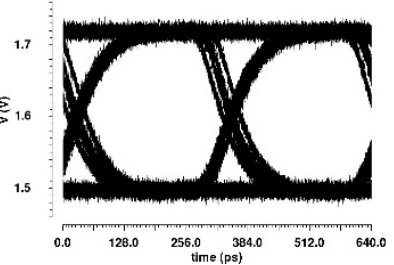

(d)
Fig. 3. Eye diagrams for $3.125 \mathrm{Gbps}$ at (a) precoder output, signal PRE, (b) equalizer input, (c) equalizer output, signal DUO, (d) decoder output, signal DEC. 